\title{
P03-11-5 Poster session
}

\section{Delays in clinical development of neurological drugs in Japan}

\section{Masayuki Ikeda}

\author{
Medical Informatics, Kagawa University School of Medicine, Japan
}

Background There was a large gap between Japan and other developed countries with regard to access to new drugs. The delays in the approval and development of neurological drugs between Japan and other countries have been a major issue for patients with neurological diseases.

Objective To analyze factors contributing to the delay in the launching of neurological drugs in Japan and to investigate effects of several important reforms in the Japanese drug approval system on the delay.

Methods We analyzed data from Japan and the US for the review and approval of neurological drugs, all of which were approved earlier in the US than in Japan, and examined the potential factors that may cause the delay of their launch.

Results Figure 1 shows the number of the 42 neurological drugs by the year of approval in Japan. Introductions of the 42 drugs in Japan occurred between December 1998 and July 2016 at a median of 87 months after introductions in the US. The mean review time of new drug applications for the 20 drugs introduced in Japan in January 2011 or later (15 months) was significantly shorter than that for the other 22 drugs introduced in Japan in December 2010 or earlier (24 months). The lag in the Japan's review time behind the US could not explain the approval delays. In the 31 of the 42 drugs, the application data package included overseas data. The mean review time of these 31 drugs (17 months) was significantly shorter than that of the other 11 drugs without overseas data (26 months). The mean approval lag behind the US of the 31 drugs (78 months) was also significantly shorter than that of the other 11 drugs (134 months).

Conclusion These results show that several important reforms in the Japanese drug development (e.g., multi-regional clinical trials) and approval system (e.g., inclusion of global clinical trial data) have substantially reduced the delays in the clinical development of neurological drugs. 